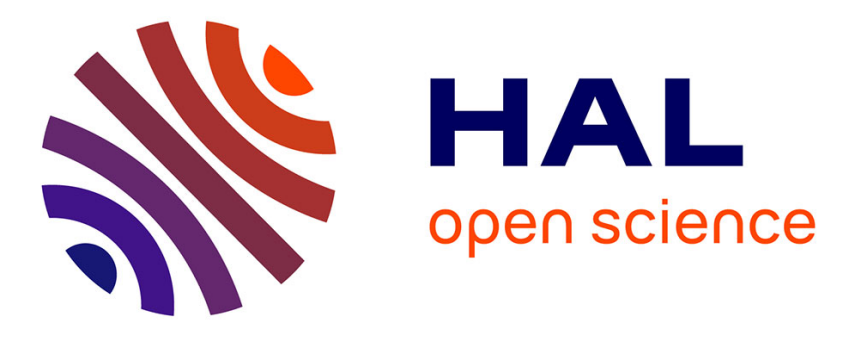

\title{
Muliebriter fremere? Le discours féminin dans les Annales de Tacite
}

\author{
Isabelle Cogitore, Louis Autin
}

\section{To cite this version:}

Isabelle Cogitore, Louis Autin. Muliebriter fremere? Le discours féminin dans les Annales de Tacite. Marion Bellissime; Pauline Duchêne. Veni, vidi, scripsi: écrire l'histoire dans l'Antiquité, 7, Ausonius Editions; UN@ Éditions, p. 103-124, 2021, PrimaLun@, 978-2-35613-379-3. 10.46608/primaluna7.9782356133793.7. hal-01806535v1

\section{HAL Id: hal-01806535 \\ https://hal.science/hal-01806535v1}

Submitted on 19 Oct 2018 (v1), last revised 2 Apr 2021 (v2)

HAL is a multi-disciplinary open access archive for the deposit and dissemination of scientific research documents, whether they are published or not. The documents may come from teaching and research institutions in France or abroad, or from public or private research centers.
L'archive ouverte pluridisciplinaire HAL, est destinée au dépôt et à la diffusion de documents scientifiques de niveau recherche, publiés ou non, émanant des établissements d'enseignement et de recherche français ou étrangers, des laboratoires publics ou privés. 


\title{
Muliebriter? Le discours féminin dans les Annales de Tacite
}

\author{
I. Cogitore \& L. Autin, UGA, Litt\&Arts - Translatio (EA 7355)
}

\section{INTRODUCTION}

\section{Accroche}

Pour Tacite, «les femmes ne peuvent et ne doivent pas être des personnages historiques » : si, par ces mots, J.-M. Engel ${ }^{1}$ cristallise ce qui paraît bien être l'opinion de l'historien-moraliste vis-à-vis des femmes de l'époque impériale, il ne rend guère compte de la complexité de la réflexion tacitéenne sur la question et, plus grave, il tombe dans le piège d'une lecture trop impressionniste de nos sources antiques, toujours très tentante avec Tacite, chez qui l'influence de la rhétorique et la multiplication des sentences donnent volontiers une image caricaturale des objets historiques étudiés. Or, la lecture minutieuse du corpus, la prise en compte exhaustive de toutes les occurrences, l'absence de quelque préjugé que ce soit, tout cela nuance rapidement le constat premier d'une misogynie à sens unique de Tacite, et nous invite à repenser la place des femmes dans le récit tacitéen. À cet égard, l'angle d'attaque du discours garantit de ne pas tomber dans des généralités, et permet du même coup de croiser les approches du corpus en mêlant analyse rhétorique et analyse narratologique.

\section{Définition du corpus}

De fait, la plupart des études qui se penchent sur le discours féminin ${ }^{2}$, voire sur le discours en général chez Tacite ${ }^{3}$, ne visent à prendre en compte que les discours suivis et les développements oratoires conséquents; à l'inverse, nous voulons nous intéresser à toutes les occurrences de la parole féminine, quelles que soient l'étendue et la modalité de ce discours, du simple verbe élocutoire au discours direct le plus long. Nous considérons que l'ensemble de ces cas forme un système significatif en lui-même. Par ailleurs, nous nous focaliserons sur les Annales, principalement parce que c'est dans cette œuvre que l'on trouve des femmes de pouvoir (certaines Julio-Claudiennes), à la différence des Histoires, qui ne présentent quasiment aucune femina réellement influente - la comparaison des femmes d'empereur entre les deux œuvres étant d'ailleurs très éclairante à cet égard. Néanmoins, nous ne nous interdirons pas d'aller chercher dans les Histoires ou dans les «opuscules » historiographiques des exemples pour éclairer notre propos.

\section{Rhétorique et efficacité}

La problématique soulevée par le discours féminin est bien sûr celle que soulève tout discours chez un historien antique, mais elle comporte aussi des questionnements spécifiques, car les femmes ont chez les historiographes une place à part, tant en raison des sources mêmes que les auteurs ont pu utiliser à leur sujet qu'en raison de la part - réelle, supposée, fantasmée - que les femmes peuvent prendre à l'action. Aussi les discours et, plus généralement, les paroles des femmes dans le récit historique doivent être questionnés avec prudence. Outre qu'il est très facile, en parlant de femmes, de se laisser aller à des généralités "genrées », aborder la question féminine sous l'angle du discours et de la parole oblige à poser de la façon la plus nette possible deux questions connexes : celle de l'exercice du pouvoir quand il s'agit des femmes de la famille julio-claudienne et celle des outils rhétoriques et de leur efficacité quand ils sont employés par des femmes.

\section{Méthodologie et problématiques narratologiques}

\footnotetext{
${ }^{1}$ J.-M. Engel, 1972, p. 301.

${ }^{2}$ Particulièrement criant chez D. Nagues, 2005. Constat fait avec beaucoup de justesse à l'échelle de l'étude des femmes en général chez Tacite par J.-L. Posadas, 1992, p. 148.

${ }^{3}$ Voir R. Utard, Le discours indirect chez les historiens latins, 2004, p. 40 (refus de considérer comme des discours indirects des passages au style indirect avec une seule subordonnée); visible aussi chez N. P. Miller, “Dramatic speech in Tacitus", 1964, et "Dramatic speech in the roman Historians", 1975.
} 
Cette étude de la ou des spécificité(s) du discours féminin chez Tacite, nous ne la voulons pas uniquement thématique : outre le contenu, la fonction et le rôle des discours, nous nous pencherons sur la forme même de ces paroles, considérant que les différentes façons d'incorporer ces voix féminines au récit historique sont signifiantes per se. Pour cette partie de notre réflexion, nous utiliserons les outils de la narratologie moderne: de fait, l'historiographie antique, de par sa dimension essentiellement narrative, fonctionne souvent selon les mêmes règles que des œuvres narratives modernes, comme par exemple le roman ${ }^{4}$. À cet égard, l'ouvrage fondamental reste Discours du Récit de G. Genette (contenu en 1972 dans Figures III). Dans le chapitre «Modes », partant d'ailleurs de l'Iliade et de la République de Platon, Genette distingue les différentes façons dont le narrateur peut rapporter des paroles qui ne sont pas siennes (« récit de paroles», p. 189) en fonction de la distance qu'elles introduisent avec le récit (p. 191-193) :

- discours narrativisé, où la parole est «traité[e] comme un événement parmi d'autres » (p. 190), par exemple «j'informai ma mère de ma décision d'épouser Albertine » (p. 191) ;

- discours au style indirect («je dis à ma mère qu'il me fallait absolument épouser Albertine», p. 191), discours «plus mimétique » (p. 192), mais où le narrateur est encore très présent ;

- discours au style direct ${ }^{5}$, où le narrateur cède (ou plutôt « feint de céder », p. 192) la parole aux personnages, et où la distance est la plus grande.

Nous faisons l'hypothèse que le choix de l'un de ces trois modes du discours rapporté n'obéit pas seulement à des visées esthétiques ou dramatiques, mais est aussi essentiellement idéologique. Malgré sa très grande richesse rhétorique ${ }^{6}$, le discours indirect reste un discours sans autonomie propre, en ce qu'il est syntaxiquement subordonné au discours narratif. Le discours direct, lui, donne à entendre ce qui se présente comme une parole non modifiée, autonome, appartenant directement à son locuteur - même si, en fin de compte, c'est bien Tacite qui réécrit les discours. Le discours narrativisé, lui, opère «la réduction du discours à l'événement » (p. 191), et correspond presque à faire entendre une parole sans contenu propre (même s'il en est fait mention). D'un de ces modes à l'autre, Tacite laisse plus ou moins parler ses personnages, et ce sont ces variations mêmes qui nous paraissent idéologiques : quel est, à cet égard, le statut du discours féminin ? Le choix de l'un de ces modes est-il lié à l'efficacité du discours, à l'ethos de la locutrice, à l'exemplarité de l'action ? Voilà ce qu'il nous faudra aussi étudier.

\section{Annonce de la progression}

Notre travail fait émerger une ligne de fracture évidente entre deux catégories de femmes chez Tacite, et ce alors que l'historien considère bien qu'il y a un comportement féminin, invariant et immuable, valable pour les femmes d'empereur tout comme pour la première affranchie venue - de la même façon que, pour Tacite, il existe un invariant comportemental pour la foule, pour les esclaves, pour les affranchis, etc. Dépassant ces affirmations sans cesse répétées, il nous faut distinguer celles que l'on pourrait appeler les femmes de pouvoir, c'est-à-dire essentiellement les membres féminins de la domus impériale des Julio-Claudiens - principalement et non exclusivement Livie, Agrippine l'Aînée, Messaline, Agrippine la Jeune et Poppée -, et toutes les autres, qu'il s'agisse de femmes d'aristocrates romains, d'affranchies, d'esclaves, de non-Romaines provinciales, voire de barbares.

\section{RHETORIQUE ET EFFICACITE CHEZ LES JULIO-CLAUDIENNES}

\footnotetext{
${ }^{4}$ Les travaux d'I. de Jong montrent d'ailleurs bien l'adaptabilité de ces outils au corpus antique (épopée, historiographie, poésie dramatique).

${ }^{5}$ L'on peut faire ici l'économie du discours indirect libre, dont l'existence, en latin, est source de débats dépassant le cadre de cette intervention.

${ }^{6}$ Mise en avant par R. Utard, op. cit.
} 
Les discours et paroles des femmes de la dynastie julio-claudienne font partie des « grands moments » des Annales et il est difficile de ne pas se laisser influencer par le statut particulier de ces morceaux de bravoure. Je me propose de considérer, femme par femme, le rapport que leurs paroles et discours entretiennent avec la rhétorique, d'une part sur le plan de l'organisation du discours, d'autre part sur le plan de l'efficacité. Examiner ces discours et paroles sur la base d'une analyse pratique a l'intérêt d'éviter les généralités, toujours dangereuses et plus encore quand il s'agit de femmes.

\section{Livie}

Commençons par Livie, bien présente dans les quatre premiers livres des Annales, et désignée d'abord comme Liuia, puis rapidement comme Augusta, titre qui résume sa place exceptionnelle de veuve d'Auguste, adoptée par testament dans la gens Iulia.

Ses paroles sont assez rares dans les Annales mais dès le début de l'œuvre, un conseil de Sallustius Crispus signale qu'elles ont un poids considérable: 1.6.3. Le contexte est celui de l'exécution d'Agrippa Postumus, selon Tacite sur ordre de Tibère et Livie ; les éventuelles paroles de Livie amèneraient la révélation de ces secrets sur lesquels repose le pouvoir de Tibère. Comme on le voit, il s'agit ici, pour ainsi dire, de paroles « en creux », puisque non prononcées par Livie ; mais nous en retiendrons l'importance, l'efficacité crainte par Sallustius Crispus : les paroles de Livie ont un poids hors du commun, leur efficacité est une évidence pour un homme de cour et de pouvoir comme Sallustius.

Or, si on considère les interventions de Livie dans les Annales, on constate qu'elles se résument à trois types de prise de paroles : des sermones, des prières (preces) et des paroles qui correspondent à une autre posture, celle de la conseillère.

Les sermones, c'est-à-dire les paroles les moins définies, sont celles que Livie échange avec Plancine, la femme du gouverneur Pison, avec qui elle agit contre Agrippine et ses enfants ${ }^{7}$. Ces paroles sont secrètes, « secretos sermones » (2.82.1). Cet adjectif, jamais innocent chez Tacite, signale l'intervention de l'historien, qui a « reconstitué » des entretiens dont il n'a pas eu la preuve, mais qu'il déduit de l'enchaînement même des faits : dans le passage 2.82, la répétition de l'adverbe ideo, repris et résumé par "hoc egisse secretos sermones » souligne l'efficacité de ces conversations secrètes, grâce à la figure de style de l'usteron proteron qui place la conséquence avant la cause. Là encore, c'est accorder à Livie une efficacité dans la parole semblable à celle que craignait Sallustius Crispus.

La plupart des paroles de Livie dans les Annales sont des paroles échangées avec Plancine, comme on vient de le voir, ou à propos de Plancine ; car cette confidente de l'impératrice, impliquée dans le procès fait à son mari pour avoir provoqué la mort de Germanicus, fait l'objet de prières adressées par Livie à Tibère pour obtenir qu'elle échappe à la condamnation. Les prières, preces, qui reviennent comme un leitmotiv dans le début du livre 3, représentent ainsi le moyen d'action que Tacite prête à Livie, conformément en somme au mode d'action qui est celui des femmes ${ }^{8}$. De plus, ces prières sont secrètes, selon Tacite : 3.15.1. La conclusion qui vient immédiatement à l'esprit est que, pour l'historien, les femmes agissent dans l'ombre, manigancent et interviennent par des voies détournées dans la politique, toujours dans le sens le plus négatif... Pourtant, réfléchissons et dépassons ce premier stade : à qui Livie adresse-t-elle ses prières ? Non pas au Sénat qui est pourtant en charge du procès de Pison, mais à Tibère, qui porte ensuite cette parole de Livie en faveur de Plancine : "pro Plancina cum pudore et flagitio disseruit, matris preces obtendens »(3.17.1). L'attitude que l'historien prête à Tibère teinte de couleurs négatives les prières de Livie, perçues comme une ingérence, une action féminine inadaptée. Or, le Senatus Consultum de Cn. Pisone patre nous propose un autre éclairage ${ }^{9}$; on y lit, 1.113 , que Tibère s'est fait l'écho des demandes maternelles

\footnotetext{
${ }^{7}$ Autre exemple 2.34.4 Livie chargeant Plancine d'agir contre Agrippine : «Plancinam haud dubie Augusta monuit aemulatione muliebri Agrippinam insectandi ».

${ }^{8}$ Sur l'efficacité supposée des prières, voir 1.13.6 : Haterius, un sénateur dont Tibère se méfie, demande à Livie de le protéger par ses prières, «curatissimis precibus »

${ }^{9}$ A. Caballos, W. Eck, F. Fernandez, 1996.
} 
: "pro Plancina rogatu matris suae deprecari ». Il ne s'agit pas ici de prières, mais de demandes, portées par un verbe, rogare, qui appartient au vocabulaire institutionnel. La comparaison des Annales et du SCPP met en lumière la démarche de Tacite, réduisant l'action de Livie à des prières, à un niveau personnel et même secret ${ }^{10}$, tandis que le $\mathrm{SC}$ désigne cette intervention de manière neutre (voire élogieuse puisque suit un éloge de Livie) et que Tibère, en relayant cette demande, agit conformément à la pietas attendue envers une mère (1.118/119 : «erga matrem suam pietati »). Les prières que Tacite prête à Livie sont donc une façon négative de présenter une action qui, dans le SCPP, n'a rien de négatif. Le point commun aux prières de Livie, dans le SCPP et dans les Annales, est qu'elles ont une efficacité, certes indirecte puisque leurs conséquences passent par le relais de Tibère, mais reconnue tant par le Sénat que par Tacite.

Enfin, le troisième de type de paroles que Tacite met dans la bouche de Livie sont des reproches adressés à son fils, 4.57.5, reproches et réclamations qui auraient, selon Tacite, poussé Tibère à quitter Rome pour la Campanie. Ces reproches et réclamations s'appuient sur l'adoption de Tibère qu'Auguste aurait accepté de prononcer devant les pressions de sa femme : "idque Augusta exprobrabat, reposcebat ». Le verbe exprobrare, quoiqu'il puisse avoir un sens technique rhétorique, renforcé par le verbe exposcere, lui-même appuyé par le préverbe ex et la valeur fréquentative du suffixe, doit être pris ici dans un sens pour ainsi dire familial : la figure de Livie est ici celle de la Mère qui gourmande un enfant, celle qui détient une autorité en tant que femme ${ }^{11}$ (et en tant que mère, puisqu'elle serait à l'origine de l'adoption). Mais Tacite est subtilement allusif : que réclame Livie à Tibère ? Rien dans le contexte de cette phrase ne permet de répondre à la question. Il faut plutôt considérer que les verbes exprobrare, exposcere sont là pour définir une attitude constante de Livie et non une action ponctuelle. À vrai dire, on peut même considérer qu'il n'y a pas ici de véritables «paroles » derrière ces verbes: Tacite résume l'attitude qu'il prête à Livie par des verbes qui expriment une forme de prise de parole, la réclamation et le blâme, mais sans en donner d'exemple. Il s'agit pour ainsi dire de paroles non prononcées, mais dont l'historien a besoin pour dessiner le portrait de la mère dominatrice, peut-être pour que Livie soit une image anticipée de ce que sera Agrippine la jeune dans son rapport à Néron. Ces «non-paroles» sont, dans le récit tacitéen, supposées avoir eu une efficacité pour ainsi dire contre-productive, puisqu'elles n'auraient rien apporté à Livie, et même, au contraire, puisqu'elles auraient provoqué le départ de Tibère pour la Campanie.

En résumé, les paroles de Livie, quand elles sont efficaces, provoquent des actions de Tibère, qui peuvent parfois ne pas apporter de résultats positifs pour elle; mais toutes les paroles contribuent au portrait d'une mère, active dans ses réseaux de clientèle féminine, et exerçant sur son fils un pouvoir considérable.

\section{Agrippine I}

Voyons maintenant ce qui concerne Agrippine l'Aînée, personnage à qui Tacite fait une part plus grande, en partie sans doute parce qu'elle est la femme de Germanicus ${ }^{12}$. Sous Tibère, la parole d'Agrippine se voit accorder une efficacité ambiguë, crainte mais non réelle.

Sa première prise de parole, 1.40.3, est brève et au discours indirect, dans le moment où son époux Germanicus face à la révolte des légions, décide d'éloigner sa femme et ses enfants. On reconnaît cependant deux parties d'un discours sans doute plus développé : une première partie sur les origines familiales d'Agrippine et une deuxième tournée vers le futur proche : "cum se diuo Augusto ortam // neque degenerem ad pericula ltestaretur ». Dans les deux parties, le vocabulaire familial,

\footnotetext{
${ }^{10}$ Comme les prières de Livie à Auguste pour qu'il adopte Tibère, 4.57.5.

11 On notera l'apparition de reproches venant d'une autre origine familiale, plus éloignée et donc moins autorisée: 2.50.1: "et Appuleiam Varillam, sororis Augusti neptem, quia probrosis sermonibus diuum Augustum ac Tiberium et matrem eius inlusisset Caesarique conexa adulterio teneretur, maiestatis delator arcessebat $»$.

${ }^{12}$ Pour un réexamen critique de la figure de Germanicus, I. Cogitore, «Tacite et Germanicus, les choix de la mémoire », Cahiers Glotz, XXIX, 2013, 157-174.
} 
celui de la légitimité (ortam, degener) fonde les arguments, dans un rapport de logique : parce qu'elle descend d'Auguste, elle ne manquera pas au devoir familial (qui implique de rester auprès de Germanicus). On a bien l'impression qu'ici Tacite résume un discours plus long, construit et argumenté selon les règles de la rhétorique. Ce sont bien là des arguments d'un discours appartenant au genre suasoire, destiné à obtenir un résultat, donc tourné vers l'efficacité. Mais l'efficacité est nulle, comme le signale Tacite avant même d'en donner le contenu: "aspernantem uxorem cum testaretur », et Germanicus éloigne sa femme.

On peut rapprocher de ce discours une autre prise de parole par Agrippine, en 1.69.2, quand elle s'adresse aux légions. Cet épisode est intéressant car il présente deux éclairages, l'un par Tacite (Agrippine agit auprès des soldats), l'autre par Pline que cite Tacite (Agrippine adresse aux soldats des éloges et des remerciements). Tout l'épisode tourne autour de l'interprétation de cette position d'Agrippine auprès des légions; sans doute Tacite lui a-t-il accordé une importance particulière, puisqu'il a tenu à signaler, ce qui est assez rare, une des sources, sans reprendre exactement ce qu'il y a trouvé : son Agrippine agit, celle de Pline parle ; mais il rappelle ces paroles, définies selon leur type rhétorique, «laudes et grates », c'est-à-dire des paroles prononcées post euentum, et non des paroles d'encouragement, non des paroles qui viseraient une efficacité. Or, toute la réflexion que Tacite prête ensuite à Tibère porte sur le danger que représente Agrippine, définie comme plus puissante que les légats et les chefs militaires, "potiorem iam apud exercitus Agrippinam quam legatos, quam duces ». C'est bien à propos de l'efficacité d'Agrippine que naissent ses inquiétudes ${ }^{13}$. Ainsi, même si les paroles sont rapportées par Pline, leur efficacité est reconnue par Tacite, ou plutôt par Tibère. L'empereur, de manière constante, prête aux paroles d'Agrippine une grande efficacité, qui l'inquiète, comme en 4.17.2: il s'agit cette fois d'un autre type de paroles, prières ou menaces, mais qui ont eu pour résultat de faire figurer Nero et Drusus, fils de Germanicus, dans les prières officielles. Tibère interprète ce résultat comme une manœuvre des partisans d'Agrippine. Efficacité, donc, mais pour laquelle on soulignera un point important : les pontifes ,sur qui la parole d'Agrippine aurait été éfficace, sont de la parenté de Tibère, donc membres de la domus au sens large ; c'est dire que l'action d'Agrippine reste inscrite dans le cercle familial.

Un épisode, précisément inscrit dans les affaires familiales, voit la parole d'Agrippine sous un jour différent: à l'occasion d'une accusation contre Claudia Pulchra, cousine d'Agrippine, celle-ci tente d'intercéder auprès de Tibère (4.52). Les paroles d'Agrippine, au discours indirect, sont soigneusement mises en scène : Agrippine est d'abord caractérisée par son ardeur excessive, semper atrox; un petit récit la peint allant trouver Tibère en train de sacrifier au Divin Auguste : contexte nettement dynastique, donc. Le discours, relativement long, comprend une structure rhétorique claire : argument 1, comparaison: non eiusdem..., avec chiasme mactare uitimas// posteros insectari; argument 2, a maiori : 1'esprit d'Auguste n'est pas dans des images/ il est en Agrippine (avec jeu sur effigies/imaginem ueram); argument 3, refutatio : Pulchra est un prétexte. Quelle efficacité pour ces paroles? Certes, elles provoquent une réaction de Tibère, qui lui répond par une maxime grecque, portant sur le pouvoir; mais elles ne provoquent aucun changement au destin de Pulchra, qui est condamnée. Que tirer alors de ce discours, si soigneusement présenté par Tacite ? 1.: C'est un discours familial, dynastique ; 2. : il a une valeur dramatique et littéraire ; 3. : il n'a pas d'efficacité concrète. Tout se passe donc comme si les paroles d'Agrippine n'avaient eu d'autre effet que de produire de la parole, la citation grecque prononcée par Tibère.

Pas plus d'efficacité pour les autres paroles d'Agrippine juste après dans le récit, concernant son éventuel remariage, 4.53.1 : là aussi, le contexte est dynastique (on sait l'importance du mariage des femmes capables de donner naissance à des héritiers), là non plus, le discours n'est pas efficace. La comparaison nous permet de voir qu'Agrippine est, là encore, caractérisée avant de prendre la parole, peruicax irae; il y a aussi une petite mise en scène (sa maladie, la visite de Tibère); aux

\footnotetext{
${ }^{13}$ La crainte qu'Agrippine puisse obtenir des résultats politiques par ses lamentations est aussi exprimée par un partisan de Pison, lorsqu'il engage ce dernier à rentrer à Rome avant les cendres de Germanicus : $2.77 .3:$ : an festinamus cum Germanici cineribus adpellere, ut te inauditum et indefensum planctus Agrippinae ac uulgus imperitum primo rumore rapiant? est tibi Augustae conscientia, est Caesaris fauor, sed in occulto, et perisse Germanicum nulli iactantius maerent quam qui maxime laetantur ».
} 
larmes silencieuses du début succèdent des prières en guise d'exorde. Le discours est, lui aussi, construit, sur le modèle d'un discours appartenant au genre suasoire : argument 1. : elle demande un mari ; argument 2. : elle est fertile ; argument 3 : cela représente un danger.

Mais là encore, les paroles n'ont aucune efficacité, même pas sur le plan de la parole puisque Tibère se tait.

Le discours d'Agrippine est donc caractérisé de manière ambiguë, par une efficacité limitée, mais qui suscite l'inquiétude quand elle sort du domaine purement dynastique : en somme, la parole d'Agrippine respecte les codes de la femme romaine, qui peut s'exprimer au sein de sa famille, mais dont le champ d'action ne doit pas excéder celui de la famille. Le problème, dans son cas, est que sa famille est la famille impériale et que les limites entre le privé et le public y sont problématiques.

\section{Messaline}

Sous le règne de Claude, le tableau diffère et les femmes y occupent une place bien davantage marquée par l'efficacité, mais de manière problématique toujours.

Ainsi Messaline, dont la parole, dans un premier temps du récit, est marquée par l'efficacité ; lors du procès de Valerius Asiaticus (11.2.3 ${ }^{14}$ ), Messaline qui convoite les jardins d'Asiaticus sur les pentes du Pincio donne à Vitellius le conseil de ne pas laisser l'accusé échapper à la condamnation. Par le verbe monet, Tacite attribue à Messaline une parole efficace, exprimant un rôle de conseillère et d'instigatrice dans un domaine où elle ne peut agir directement, puisqu'elle n'a pas le pouvoir de condamner (contre Poppaea en revanche, avec qui Asiaticus aurait eu des relations adultères, elle agit de façon à la pousser au suicide). Cette parole efficace place Messaline dans une situation qui ressemble à celle de Livie, la situation de la femme conseillère. Toutefois il faut souligner que celui sur qui sa parole est efficace est Vitellius et non Claude : sa parole sort donc du domaine familial et a une action plus large. On peut considérer que c'est ici le sommet de l'action de Messaline, le moment où sa parole est le plus efficace, et sort précisément du cercle familial, dans un fonctionnement presque masculin. Elle est l'expression du fonctionnement d'un réseau d'appuis politiques, dont Vitellius fait partie à ce moment. La situation change ensuite. En effet, quand elle prend à nouveau la parole, c'est au moment où sa chute est proche : elle s'adresse alors à Vibidia, la plus âgée des Vestales, pour que celle-ci sollicite la clémence du grand pontife (11.32.5); ici la parole de Messaline passe par la prière, orauit, et constitue une demande d'intervention; on notera également que cette demande est adressée à une femme : on peut ainsi dire que, à ce stade de son histoire, Messaline en revient à des paroles de type féminin, des prières et des demandes d'intervention dans un cercle limité, ici un cercle féminin. Lorsqu'elle tente de s'adresser à un cercle plus large, ce qu'indique le verbe clamitare, elle n'est pas entendue, sa voix est couverte par celle de l'accusateur (11.34.3). Il ne lui reste que les plaintes et les larmes ${ }^{15}$ : précisément, ce type de paroles est sans efficacité aucune : 11.37 .6 : «lacrimaeque et questus inriti ducebantur ».

La parole de Messaline est donc en quelque sorte représentative de son action, d'abord transgressive dans sa relation à son amant Silius, puis revenant en des bornes plus féminines quand la chute est proche.

\section{Agrippine II}

Agrippine, la dernière femme de Claude, est une des figures féminines les plus travaillées dans les Annales et cela est visible dans son discours. Tacite lui prête des paroles de nature variée : on trouve ainsi des demandes comme lorsqu'elle obtient, impetrat, de Claude le retour d'exil de Sénèque (12.8.4) ou quand elle obtient (même verbe, impetrat) une déduction de colonie chez les Ubiens en Germanie (12.27.1). Sa parole est efficace en ce qu'elle provoque une décision de Claude, dans la position plus forte que celle de conseillère, le verbe impetrare exprimant une volonté impérieuse.

\footnotetext{
14 «Intra cubiculum ».

${ }^{15}$ Et par un libelle, un écrit...
} 
Efficacité aussi lorsque Britannicus, après l'adoption de Néron par Claude, persiste à l'appeler par son nom de Domitius, provoquant la colère d'Agrippine, qui s'adresse à Claude 12.41.7 : "quod ut discordiae initium Agrippina multo questu ad maritum defert: sperni quippe adoptionem, quaeque censuerint patres, iusserit populus, intra penatis abrogari ; ac nisi prauitas tam infensa docentium arceatur, eruptura in publicam perniciem». Le discours est repris au style indirect, caractérisé d'abord par le ton de la plainte, «muto questu », précisé par le verbe defert, verbe « officiel » pour les dépôts de plainte. Or le contenu du discours est donné, immédiatement après (sans doute faut-il voir dans les «plaintes » ce qui a pu être l'exorde du discours); argument 1, argument de droit : une décision officielle, l'adoption, est méprisée dans la pratique privée; argument 2, politique et de précaution : il faut maîtriser cela sous peine de mettre en danger l'État. Le discours est construit de manière raisonnée, finissant par l'argument le plus grave, avec un conseil de passage à l'action formulé indirectement, "nisi arceatur », qui n'empêche pas de considérer qu'il s'agit d'un discours appartenant au genre suasoire. L'efficacité du discours est immédiate, puisque Tacite enchaîne par le participe passé passif commotus, et la décision que Claude prend conformément à la volonté d'Agrippine.

Autre type de paroles chez Agrippine, mais même efficacité, dans d'autres contextes : quand elle fait passer le commandement des cohortes prétoriennes à Burrus (12.42.2: «adseuerante uxore // Transfertur regimen»: la conséquence est immédiate) ou quand elle obtient qu'un accusateur de Vitellius son partisan soit exilé, 12.42.5. Dans ce passage, la précision que donne Tacite a tout son sens : Agrippine obtient cette décision "minis magis quam precibus » : on est ici loin des prières vaines d'une femme désarmée.

Mais la situation change dès l'avènement de Néron, dans un paysage où Tacite se plait à rappeler le rôle du Sénat, conforme à ce qu'on peut en espérer. Des mesures que Néron soumet au vote des sénateurs et auxquelles Agrippine s'oppose passent quand même: 13.5.1, "aduersante Agrippina » signale une parole, sur laquelle Tacite donne quelques informations ; la scène se passe au palais impérial, dans la bibliothèque du temple d'Apollon Palatin, lors d'une séance du Sénat à laquelle Agrippine assiste cachée derrière un rideau. Tout ici est marqué par le goût de Tacite pour la mise en scène politique et la réflexion sur le rôle des femmes. Mais si on essaie de s'interroger concrètement sur cette scène, il faut imaginer que Néron aurait proposé aux sénateurs de voter ces mesures sans en avoir au préalable parlé avec sa mère ; imaginer encore que celle-ci, depuis sa cachette, aurait pu faire savoir à Néron son opposition, puisque l'expression «tamquam acta Claudii subuerterentur» contient l'argument avancé par la mère de l'empereur. Comment l'aurait-elle fait savoir à Néron ? Comment le souvenir s'en serait-il perpétué, puisqu'il est peu probable que ces paroles aient été discutées en séance du Sénat? Bref, toutes ces interrogations nous amènent à souligner à quel point la parole prêtée à Agrippine est ici le résultat d'une construction du récit tacitéen. Comme nous sommes là aux tout débuts du règne de Néron, c'était pour Tacite l'occasion de commencer à décrire la chute progressive d'Agrippine et sa perte d'influence, la perte d'efficacité de cetta parole qui, sous Claude, avait eu du poids.

Mieux encore, c'est en montrant que sa parole finit par être contre-productive que Tacite peint cette chute. En 13.13.1 ses paroles s'accumulent, sans qu'il soit ici question de discours suivi, mais plutôt d'une logorrhée féminine, muliebriter: ces paroles, dont le ton est celui du reproche, exprobrare, au lieu d'obtenir le résultat attendu, poussent Néron dans la voie inverse, l'écarte de la pietas et de l'obsequium envers sa mère.

Tacite prête alors à Agrippine un assez long discours, au moment où le choc avec son fils est devenu patent, 13.14.2. Comme on l'a vu déjà ailleurs, Agrippine est d'abord caractérisée, ici par l'adjectif praeceps et le verbe "ruere ad terrorem et minas »: c'est le signe d'un discours violent, probablement sans exorde une fois encore. L'examen des arguments et de la construction de ce discours fait apparaître que le premier argument, qui repose sur des constatations dynastiques, puisque concernant Britannicus, surprend: Agrippine en effet y affirme la légitimité de Britannicus, dans un 
revirement total par rapport à son attitude antérieure ${ }^{16}$. Argument $2:$ Agrippine menace de tout dire sur la famille : ici, la formulation par une litote, "non abnuere quin ...patefierent » attire l'attention du lecteur, qui peut voir en Agrippine la figure inversée de Livie, à qui il était conseillé de ne pas divulguer les arcana dans une autre affaire familiale, celle d'Agrippa Postumus. Il est, de cette façon, tout à fait clair que la parole féminine impériale, si elle est dévoilement, est dangereuse ; la menace du dévoilement est la plus sérieuse, et constitue un des rares actes que pouvaient commettre les femmes. C'est dire de façon irréfutable que leur parole est efficace et dangereuse. De fait, l'argument suivant, avec la forme verbale au futur, ituram 〈esse>, est une précision dans la menace. Enfin, la fin du discours, décrite et caractérisée mais non reprise en discours indirect, consiste en l'accumulation de tons et d'arguments, adgerere probra, qui reprend le thème du blâme, et inuocare qui représente l'appel aux divinités. Pour ce discours, Tacite a particulièrement travaillé son matériau, choisissant certains arguments pour les inclure dans le discours indirect, en gardant d'autres pour cette accumulation finale; dans l'évolution vers un climax dramatique, le discours joue un rôle fondamental.

Le discours suivant, 13.21.2, est cette fois le discours direct qu'Agrippine prononce pour se disculper de l'accusation de vouloir mettre Rubellius Plautus sur le trône. Après la caractérisation, "ferociae memor », Tacite fait commencer le discours sans exorde, brusquement, "non miror », par une accusation contre Silana, une femme qui aurait suscité les accusateurs contre Agrippine; le premier argument est donc directement une refutatio ; la deuxième partie du discours, 13.21.3, "nam Domitiae inimicitiis gratias agerem ", est une accusation contre la tante de Néron, développée en 13.21.4. L'argument suivant, ab absurdo, vient clore le raisonnement par une finesse qui permet à Agrippine d'affirmer son amour pour son fils, d'une manière qui peut contenir des menaces (menaces de révéler ce qu'elle a fait pour son fils, comme en 13.14.2).

La comparaison de ces deux discours successifs est éclairante : on voit bien qu'en réalité, ils pourraient ne former qu'un seul et même discours, fondé sur des arguments familiaux (place de Britannicus, de Rubellius Plautus et leur légitimité respective), sur la menace de divulguer les dessous de l'accession de Néron au pouvoir grâce à sa mère. La grande variété des tons, blâme, invocation, réfutation, accusation, menaces, est le signe d'un matériau littéraire riche, dans lequel Tacite a pu puiser et qu'il a pu répartir en variant ses effets, par le style indirect et le style direct, très rare pour une femme. La fin de l'épisode est significative : Agrippine demande une entrevue, conloquium, avec son fils, dans lequel elle quitte le ton du blâme et obtient une satisfaction immédiate, la punition des délateurs et des récompenses pour ses amis. Ce /ces discours sont donc marqués par une efficacité immédiate... et une absence d'efficacité à moyen terme, puisqu'on sait qu'Agrippine va perdre la vie dans la prochaine action de son fils. Mais il convient de souligner la grande importance que l'historien a tenu à donner à ces paroles d'Agrippine, dans ce premier moment de crise intense.

\section{Poppée et Octavie}

Enfin, dernières femmes à prendre la parole dans les Annales, Poppée et Octavie présentent une configuration bien différente : pour Octavie, seulement quelques mots au discours indirect, situés au moment de sa mise à mort. Ses arguments reposent sur les liens familiaux, avec Néron dont elle reste la sœur après en avoir été divorcée, avec Germanicus et Agrippine, mais n'obtiennent aucun résultat. Tacite a ici choisi de souligner la tension dramatique par la description précise du processus mortel, par l'image de la tête coupée et la rapidité du récit; les paroles sont pour ainsi dire juste un écho dynastique.

\footnotetext{
${ }^{16}$ Cette attitude est déjà présente en 12.69.2 : « iam primum Agrippina, uelut dolore uicta et solacia conquirens, tenere amplexu Britannicum, ueram paterni oris effigiem appellare ac uariis artibus demorari ne cubiculo egrederetur. Antoniam quoque et Octauiam sorores eius attinuit, et cunctos aditus custodiis clauserat, crebroque uulgabat ire in melius ualetudinem principis, quo miles bona in spe ageret tempusque prosperum ex monitis Chaldaeorum aduentaret ». Le verbe appellare, fondamental, marque que cette parole reste une parole et ne se traduit pas en acte.
} 
Dans le cas de Poppée, la situation est différente et Tacite lui donne la parole assez souvent, surtout par rapport à la brièveté de son "passage » dans le récit. Un premier discours, 13.46, est destiné à pousser Néron à écarter son époux Othon ; les arguments en sont construits : argument $1, a$ persona, elle est mariée ; argument 2, a persona de l'époux, Othon a toutes les qualités ; argument 3, $a$ persona Neronis : Néron s'avilit dans sa relation avec Actè. La conclusion n'est pas formulée, mais elle intervient sous forme de conséquence, "deicitur ... Otho », forme d'efficacité optimale. Un deuxième discours 14.1.1 lui fait suite, Poppée désirant le mariage avec Néron. Il s'enchaîne naturellement au premier par l'ablatif «crebris criminationibus », qui pourrait signaler un résumé de paroles disséminées dans le temps, tout comme dictitans dans le premier discours pouvait résumer plusieurs prises de paroles. Appartenant au genre suasoire, le discours débute abruptement par des questions, "cur enim, formam scilicet displicere », marquées par une ironie mordante ; leur font suite des menaces, «quod si ...posset, // ituram ».

Enfin, le dernier discours de Poppée, 14.61, fort long, est presque l'exact parallèle de ce dernier : caractérisées d'abord par la psychologie de Poppée, «semper odio, tum et metu atrox », les paroles, dans un discours indirect très flou (pas de verbe introducteur) reprennent des arguments déjà vus et les développent: argument 1, a persona : Poppée est en danger; argument 2, a persona Neronis, c'est Néron qui est en danger aussi, 61.3 : «arma illa aduersus principem sumpta», avec une assimilation de Néron à l'État, lui-même en danger face à un tumultus possible ; argument $3:$ retour sur la persona de Poppée, innocente, avec la même ironie que celle vue dans le précédent discours ; péroraison, marquée par denique: appel à l'action; et enfin par une menace, avec la structure désormais bien repérée de l'hypothèse, "si desperent...daturos ». En comparant les deux discours, on voit que la parole de Poppée s'est faite plus riche, que l'appel à l'action est plus net, avec expression d'un ordre adressé à Néron, acciret. Le discours est donc bien l'expression dramatique de la personne, en conformité avec l'avancement du récit. De fait, Tacite prend ici le temps de considérer l'efficacité de ce discours, qu'il définit comme uarius pour en souligner la richesse : efficacité totale sur Néron, marquée par le parallélisme des termes: "Varius sermo et ad metum atque iram adcommodatus terruit simul audientem at accendit: metum/terruit, iram/accendit». De ce fait, le récit peut s'enchaîner comme une évidence dramatique, vers la mort d'Octavie que Poppée a voulue.

\section{LES DISCOURS FEMININS AU-DELA DES JULIO-CLAUDIENNES : UN LONG IGNAUUS QUESTUS?}

Dans les Annales, et à partir de notre relevé, les Julio-Claudiennes, représentées presque uniquement par les cinq grandes figures que sont Livie, Agrippine l'Aînée, Messaline, Agrippine la Jeune et Poppée, parlent autant de fois que toutes les autres femmes réunies ${ }^{17}$; surtout, elles parlent plus massivement et leurs interventions sont régulièrement détaillées grâce à un discours indirect de plusieurs propositions. Malgré leur disparate, les autres femmes ont en commun leur impossibilité totale d'accéder, même indirectement, au pouvoir, à la fois constitutionnellement et pragmatiquement, puisque, non issues de la domus impériale, elles peuvent difficilement y faire jouer des réseaux d'influence, comme les Julio-Claudiennes le font régulièrement ${ }^{18}$. L'on s'attendrait donc volontiers à trouver, chez ces femmes, un discours sans aucune conséquence, des voix sans efficacité, réduites à leur simple dimension sonore.

\footnotetext{
${ }^{17}$ Nous relevons trente-sept discours de non Julio-Claudiennes contre quarante-et-un des Julio-Claudiennes (les cinq citées auxquelles s'ajoutent Octavie, Marcia, Domitia Lepida et Julie (une intervention chacune).

${ }^{18}$ Le cas de Boudicca est bien entendu très différent, et mériterait à lui seul une étude que le temps dont nous disposons ne nous permet pas même d'entamer : elle se présente à la fois comme femme, comme épouse, comme $d u x$ et comme issue de la foule ("unam e uolgo »,14.35). De manière générale, il semble que Tacite ait voulu teindre l'épisode du soulèvement des Bretons (14.29-39) d'une réflexion, en sous-texte, sur la place des femmes. Le discours de Boudicca est éloquent à cet égard. Un exemple parmi d'autres : le fait de médier les prodiges annonçant la guerre (en 14.32) par une voix féminine et collective ("feminae in fuorem turbatae adesse exitium canebant... ») relève d'un choix conscient - Dion Cassius, rapportant la même scène (62.1), n'a ainsi recours à aucun discours médié autre que celui du narrateur.
} 
Ce constat vaut sans équivoque pour les cas de groupes exclusivement féminins. Contrairement à ce qui a pu être écrit ${ }^{19}$, l'on trouve bien dans les Annales une dizaine d'occurrences ${ }^{20}$ de discours émanant d'un muliebre agmen (selon l'expression de Tacite en $14.30^{21}$ ). Étant à la fois voix féminines et voix collectives, ces bruits ne pouvaient guère bénéficier de l'indulgence de Tacite, et, de fait, ils se laissent aisément ranger en deux grandes catégories : cris bachiques du furor féminin ${ }^{22}$, pour lesquels Tacite reprend des topoi bien connus, ou, pour la majorité des occurrences, cris de lamentation ${ }^{23}$. Les termes renvoyant à ces discours sont d'ailleurs fort significatifs : lamenta (trois fois), souvent suivi du génitif coniugum ou feminarum, lamentatio, lamentor, planctus, defleo, lacrimae: Tacite fait jouer tout son sens de la uariatio pour insister sur le pathétique de telles interventions. Ce pathétique est une constante pour les groupes de femmes, qu'elles soient romaines (3.10) ou barbares $(4.51)$, issues de l'aristocratie $(1.40 ; 6.10)$ ou de la plèbe $(15.38 ; 16.10)$ : c'est que, tout comme pour les foules masculines, Tacite s'intéresse moins aux variations de lieu, de statut sociopolitique ou de circonstances qu'à l'invariant comportemental de ces groupes, auquel il assigne des caractéristiques psychologiques indépassables, ici la tendance à la déploration. Cet atavisme pour la lamentation aboutit donc à des incongruités, qui servent bien sûr le caractère frappant de l'œuvre, comme ces lamentations féminines au beau milieu du champ de bataille (4.51), c'est-à-dire dans un espace dont le paysage sonore est, normalement, essentiellement constitué de clameurs masculines.

Du même coup, c'est la participation à l'action qui est interrogée pour ces voix féminines collectives. Le délire bachique ou la lamentation entrent-ils dans le champ de l'analyse des causes, qui est l'objectif premier, thucydidéen si l'on veut, de Tacite ${ }^{24}$ ? À première vue, non. De fait, la grande majorité des cas montrent ces bruits féminins complètement détachés de l'action ; ils entravent même sa progression en 15.38. De nombreux marqueurs signalent, dans le texte tacitéen, que ces femmes se situent à côté de l'action historique, et non en son cœur, notamment la récurrence de la préposition ou du préverbe $a d^{-25}$, qui fonctionne comme une indication scénique de cette adjacence à l'action des feminae. En ce sens, les groupes féminins servent plutôt à donner de leur voix un cadre sonore, souvent pathétique, à un événement, à dramatiser un tableau en lui ajoutant une dimension acoustique.

Ceux-ci, précisément, présentent une variété bien supérieure de formes et de fonctions. Là où le discours collectif féminin avait rarement une visée ou même une posture rhétorique, près de deux discours individuel sur cinq s'inscrivent dans une démarche argumentative (convaincre son interlocuteur). Même si certains extraits restent dans le champ de la pure déploration ${ }^{26}$, la coloration lexicale est généralement tout autre, à ne regarder par exemple que les termes introducteurs de ces paroles : hortari (6.35), flagitare (11.34), orare (12.51), arguere (15.51), denegare (15.57) ou encore consilium (15.54) en sont de bons exemples. De même, la tendance de ces femmes à la calomnie ${ }^{27}$ abonde dans le même sens : elles ont conscience de la puissance potentielle de leur parole.

Le cas du pathétique nous paraît fort significatif. Les groupes féminins, nous l'avons vu plus haut, semblent fréquemment utilisés par Tacite pour donner une coloration pathétique à un passage, d'une façon tout à fait lisible (on représente directement une déploration qui n'a d'autre rôle que stylistique). C'est une caractéristique presque ontologique, comme l'atteste l'expression muliebris eiulatus (utilisée pour Pollitta, femme de Rubellius Plautus, en 16.10). Chez les femmes prises individuellement cependant, le pathétique devient une arme rhétorique, conformément d'ailleurs aux principes aristotéliciens (Rhét., 2.1, 1378a et 2.2-11, 1378b-1388b) et à la rhétorique ancienne en

\footnotetext{
${ }^{19}$ J.-M. Engel, 1972, p. 291 et malgré la note 2.

${ }^{20} 1.40 ; 3.1 ; 4.51 ; 6.10 ; 14.30$ (avec des bruits rapportés en 14.35 et 36 ) ; $14.32 ; 14.60 ; 15.37 ; 15.38 ; 16.13$.

${ }^{21}$ Présente aussi en 1.40 et en 14.13 .

$2214.32 ; 15.37$.

${ }^{23} 1.40 ; 3.1 ; 4.51 ; 6.10 ; 15.38 ; 16.13$.

${ }^{24}$ Voir le texte fameux du prologue des Histoires, où Tacite affirme écrire l'histoire "ut non modo casus euentusque rerum, qui plerumque fortuiti sunt, sed ratio etiam causaeque noscantur $»(H, 1.4)$.

${ }^{25}$ Préverbe en 4.51 ( «adsistentes ») et 15.37 ( «adstabant») ; préposition en 15.38 ( ad hoc lamenta ») : les femmes doivent être testes (14.34) du déroulement historique.

${ }^{26}$ Voir surtout 12.47, à propos de la femme de Mithridate : «coniux cuncta lamentatione complebat ».

${ }^{27}$ Ainsi pour Plancine (2.55) ou pour Annia Rufilla (3.36).
} 
général. Deux de nos passages montrent très nettement l'utilisation du pathétique pour faire effet sur un auditoire précis et pour servir, de ce fait, la situation de la locutrice, souvent critique. La mère de Sextus Papinius, accusée d'inceste avec son fils (6.55), se sert du pathos pour essayer de convaincre ses juges ${ }^{28}$. De même, Aemilia Lepida, accusée d'adultère (3.22-23), se défend par une lamentatio flebilis, qui cache en réalité une argumentation cohérente et précise, essentiellement fondée sur le rappel de son illustre ascendance, c'est-à-dire sur la mise en avant d'un certain ethos : «lamentatione flebili maiores suos ciens ipsumque Pompeium tantum misericordiae permouit...». Il est assez intéressant de voir que Lepida s'entoure de femmes illustres («cum claris feminis »), qui concourent probablement à rappeler aussi sa noblesse, mais qui sont peut-être choisies pour les potentialités pathétiques du groupe féminin, pour donner un écho à la déploration de Lepida ${ }^{29}$. Quoiqu'il en soit, le pathétique est ici une arme rhétorique, et qui plus est une arme efficace, tout du moins dans un premier temps. En effet, la foule du théâtre s'émeut directement, et l'efficacité de la stratégie de Lepida est toute contenue dans la consécutive «tantum misericordiae permouit ut... clamitarent ». Le pathétique n'est donc pas vain : il fait effet sur les spectateurs, sur ce uulgus qui tait son nom ; mais cette stratégie n'empêche pas Lepida d'être condamnée à l'exil, comme Tacite l'explique très laconiquement dans la suite directe de l'extrait: " mais ensuite les esclaves mis à la torture dévoilèrent les scandales de sa vie, et on se rangea à l'avis de Rubellius Blandus qui lui interdisait l'eau et le feu » (trad. Goelzer). Toute l'ambiguïté du pathétique féminin est là : souvent efficace sur l'auditoire, il n'a presque jamais d'influence sur le cours réel des événements. Sa portée est en quelque sorte limitée à la sphère rhétorique, sans que le discours ne se concrétise en action.

C'est que, pour Tacite, il existe une véritable perméabilité entre la foule et la femme. En 1972, J.-M. Engel parlait déjà de « discrète mais constante analogie » (p. 291), sans réussir à établir d'autre lien qu'un ensemble de clichés aristocratiques pouvant servir pour les deux entités : leur caractère passionnel, leur crédulité, leur goût des intrigues (p. 303-307). Pourtant, un lien plus direct existe : une connexion se fait souvent entre la femme qui parle et la foule qui écoute précisément grâce à leur sensibilité au pathétique, que ne partagent pas nécessairement les individus, voire les groupes aristocratiques masculins (les sénateurs). Les ultima uerba de Germanicus sont ainsi largement composés d'une réfutation du pathétique comme discours féminin ou collectif et anonyme (c'est-à-dire vulgaire) au profit d'une exhortation topique à l'action ${ }^{30}$. Mais la foule, elle, fait largement écho à ce pathétique, le reçoit et, bien souvent, le reproduit. C'est très net dans l'extrait précédent (lamentation de Lepida au théâtre), dans lequel la figure stylistique dominante est le parallélisme, précisément pour marquer cette continuité entre la déploration féminine (qui est donc une stratégie rhétorique) et la déploration collective ${ }^{31}$. N'est-ce pas d'ailleurs ce lien qui est évoqué comme principal danger, si Pison, en suivant la mort de Germanicus, rentre à Rome après Agrippine ? Dans la proposition «te (...) planctus Agrippinae ac uulgus imperitum primo rumore rapi[ent] », la conjonction ac nous parait presque consécutive. Mais ce rapport presque intime n'est pas l'apanage des femmes prises individuellement : le seul moment où les voix collectives féminines influencent indubitablement le cours des choses ${ }^{32}$ passe par cette transmission du pathétique des femmes à la foule des soldats de Germanicus, pathos qui stoppe la mutinerie et les amène à se repentir auprès de leur général : le chapitre 1.40 se termine par les «lamentantes amicorum coniuges »; le chapitre 1.41 débute par la naissance d'une rumeur chez les soldats, se poursuit par leur sentiment de repentir et de commisération («pudor et miseratio ») et se termine par leur retour auprès de Germanicus. Le lien entre les deux

\footnotetext{
28 «Quamquam (...) alia in eundem dolorem maesta et miseranda diu ferret ».

${ }^{29}$ Peut-être la même stratégie est-elle utilisée par Libo Drusus lorsque, accusé de fomenter une révolution, il s'entoure des primores feminae pour susciter le pathétique chez ses amis (2.29).

${ }^{30}$ «Non hoc praecipuum amicorum munus est, prosequi defuctum ignauo questu, sed quae uoluerit meminisse, quae mandauerit exequi. Flebunt Germanicum etiam ignoti : uindicabitis uos (...). Ostendite populo Romano diui Augusti neptem eandemque coniugem meam » (2.71). Voir aussi, à propos des Germains, "feminis lugere honestum est, uiris meminisse » (Germ., 27).

${ }^{31}$ Voir aussi la continuité entre la déploration de la femme de Mithridate et celle qui assistent à la déchéance du roi (12.47 : «coniux cuncta lamentione complebat » suivi de « erant contra qui miserarentur »).

${ }^{32}$ Voir aussi le rôle des lamentations des femmes thraces, qui augmentent le courage des combattants (4.51) ou, pour quitter le pathétique, les prophéties des Icéniennes, qui poussent les Bretons à l'action (14.32).
} 
groupes est explicite: "gemitus ac planctus 〈coniugum> etiam militum auris oraque aduertere » (2.41).

L'on voit bien ici toute l'ambiguïté du discours pathétique féminin, qui constitue la majeure partie du discours collectif et une part importante des discours individuels : il n'est certes pas dépourvu d'efficacité, mais celle-ci se limite ordinairement à une impression sur la foule, qui réagit par mimétisme, à un effet qui laisse rarement sa marque dans le cours des événements. Les Thraces ont beau être stimulés par les lamentations de leurs épouses, ils seront rapidement amenés à résipiscence par Poppeus Sabinus ; la démonstration pathétique d'Aemilia Lepida ne l'empêchera pas d'être condamnée ; Tacite est encore plus explicite lorsqu'il intègre le discours féminin pathétique dans des structures concessives, notamment avec quamquam ${ }^{33}$. Pathos inefficace, donc, désordre passionnel bien inférieur, aux yeux du moraliste, à l'ordre rationnel; mais cette utilisation du pathétique féminin reste équivoque, et il n'est bien sûr pas interdit de substituer à cette foule intradiégétique, sensible au discours de lamentation féminin, le uulgus extradiégétique qui sert de public (l'un des sens de uulgus) à l'historien : cette porosité entre la femme et la foule fait aussi le jeu de l'écrivain-dramaturge qu'est Tacite.

Les discours féminins individuels ne se cantonnent toutefois pas au pathétique, loin de là. Comme on l'a dit plus haut, beaucoup s'engagent dans des postures rhétoriques, visant ainsi à convaincre son auditoire et à se traduire en action. Néanmoins, leur réussite demeure largement problématique : moins d'un discours féminin (individuel ou collectif) sur cinq n'est pas suivi d'effets concrets sur la trame historique, quand la même proportion ne parvient qu'à susciter une émotion (déploration ou indignation) sur son auditoire sans autre conséquence, la majorité des discours (plus d'un sur deux) n'ayant même aucune influence sur les interlocuteurs de l'oratrice et sur les événements. La différence est frappante avec Julio-Claudiennes, chez lesquelles plus d'une intervention sur deux est suivie d'effets concrets. Il nous reste à voir si la forme de ces discours marque cette différence.

\section{DE LA FORME DES DISCOURS COMME OUTIL HISTORIOGRAPHIQUE}

Chez les historiens anciens, le récit ne peut être simplement considéré comme le miel de Lucrèce : il ne s'agit pas d'une forme distrayante tout à fait dissociée de la leçon historique, mais bien plutôt d'un outil historiographique signifiant, à analyser comme tel, notamment grâce aux outils de la narratologie moderne. L'on peut ainsi se demander ce que transcrit le choix du discours narrativisé, indirect ou direct pour rapporter les discours féminins - c'est-à-dire l'autonomie plus ou moins grande donnée à la voix féminine.

Le premier constat à faire est la rareté des discours directs féminins. Alors que les hommes s'expriment chez Tacite alternativement en discours directs et en discours indirects, les femmes, elles, ne jouissent que très rarement du premier : on en dénombre très exactement six dans les Annales. Quatre sont à attribuer à Agrippine la Jeune : un véritable discours suivi (13.21), et trois brèves sentences rapportées au moment de sa mort et fort célèbres - le blâme adressé à la servante qui l'abandonne ( "tu quoque me deseris », qui fait penser aux ultima uerba de César, en 14.8), la bravade symbolique à Anicetus qui vient la tuer ( «uentrem feri », ibid.) et la réponse pleine de fierté faite à la prophétie des Chaldéens selon laquelle son fils la tuerait («occidat, dum imperet», en 14.9, qui semble cette fois faire écho au vers d'Accius, emblématisé par Caligula). Outre ces quatre passages, vient ensuite le discours direct surprenant de Servilia lors du procès de son père Soranus (16.31), et enfin un cas qui ne rentre pas réellement dans notre corpus, puisqu'il s'agit d'une phrase prophétique prononcée par une uates, elle-même inspirée par la divinité, Tacite parlant d'ailleurs non de mulier, mais de species muliebris.

\footnotetext{
${ }^{33}$ Ainsi pour la mère de Sextus Papinius, accusée d'inceste : "quamquam genua patrum aduolueretur luctumque communem et magis imbecillum tali super casu feminarum animum aliaque in eundem dolorem maesta et miseranda diu ferret » (6.55).
} 
Si l'on excepte le cas d'Agrippine, un peu particulier (nous y reviendrons), les femmes sont globalement privées d'une parole directe : au mieux, leur discours est reformulé par la voix narrative et syntaxiquement enclos et dépendant d'elle (discours indirect); sinon, leur discours est réduit à un terme, verbe ou substantif, et traité comme un événement parmi d'autres (discours narrativisé). Cette première particularité prolonge le parallèle entre la femme et la foule, à l'exception qu'il n'existe absolument aucun cas de discours direct rattaché à un groupe ${ }^{34}$ : les collectifs s'expriment essentiellement de manière médiée, ils ont besoin de l'autorité du narrateur pour reformuler leurs propos. Voilà un élément narratologique qui nous paraît constitutif d'un « discours dominé », qui textualise, en quelque sorte, la position politique des femmes à Rome, tout comme des foules.

Au-delà de ce premier constat, le choix du type de discours ne nous paraît pas avoir la même valeur pour les femmes proches du pouvoir (les Julio-Claudiennes) que pour les autres. Commençons par ces dernières. L'utilisation d'un discours plutôt qu'un autre nous paraît corrélée pour ces femmes à deux dynamiques : celle de l'efficacité et celle de l'exemplarité des paroles. L'observation précise des différentes interventions féminines tend à montrer que l'utilisation du discours indirect (voire du discours direct) par rapport au discours narrativisé, c'est-à-dire d'une parole plus autonome par rapport à une parole réduite à sa dimension événementielle, est liée à la volonté de mettre en avant l'originalité d'un discours féminin, parce qu'il laisse sa trace dans l'Histoire ou parce qu'il délivre un modèle à suivre pour le lecteur de Tacite.

C'est particulièrement clair pour les discours collectifs féminins. Ceux-ci sont, pour la plus grande partie (75\%), dépourvus de toute conséquence: aussi sont-ils sensiblement proportionnellement $(90 \%)$ des discours narrativisés, dont le contenu n'est qu'esquissé ; inversement, le seul discours indirect rattaché à un groupe féminin - les prophéties des Icéniennes, en 14.32 - est aussi un des rares à avoir une conséquence, certes indirecte (pousser les Bretons à la guerre).

Les discours de femmes non julio-claudiennes amènent aux mêmes conclusions, même si celles-ci doivent être nuancées. Ainsi, leurs discours narrativisés sont globalement des discours inefficaces qui n'influencent rien, sinon, parfois, l'esprit des auditeurs ; rares sont ceux qui débouchent sur une action excédant le cadre énonciatif (moins de 15\%).

Les discours indirects permettent aussi de rendre compte de paroles qui pèsent plus dans le cours de l'Histoire. Ce n'est pas tant une question de longueur - certains discours indirects sont plus brefs que des discours narrativisés, où plusieurs verbes déclaratifs sont parfois coordonnés - mais bien une posture idéologique: en laissant entendre, quoique de manière médiée, la voix féminine, en ouvrant un espace pour son expression semi-autonome, en stoppant l'enchaînement du récit pour ménager une pause discursive, Tacite textualise leur position plus marquée dans l'étude des causes. Si l'on veut d'autres preuves que statistiques, le comportement de la femme de Milichus est assez éloquent (15.54). Milichus, affranchi de l'un des principaux membres de la conjuration de Pison, Flavius Scaevinus, en cause la perte en la révélant à Néron. Mais c'est bien sa femme le véritable moteur de cette décision. Celle-ci, jamais nommée, apparait comme une influence presque annexe ( "etenim uxoris quoque consilium adsumpserat », à la toute fin du chapitre 15.54), aussi son discours est-il d'abord narrativisé («quippe ultro metum intentabat»); mais au fur et à mesure que Tacite dévoile à quel point ce simple consilium a de force sur Milichus, son discours, dans l'emballement du récit, devient indirect - la syntaxe très libre de cet extrait, qui coordonne un discours narrativisé et un discours indirect dans un effet de zeugme assez surprenant ${ }^{35}$, insistant peut-être sur ce rôle soudainement devenu central de la femme. Cette parole ayant soudainement acquis un véritable poids dans le récit historique, il est normal que Tacite en marque nettement la conséquence au début du chapitre suivant ("igitur »,15.55). Outre l'évidence de la dichotomie entre le rôle de la femme dans l'épisode et la réticence que Tacite a à le reconnaitre, l'on voit bien ici la corrélation qui se met au jour entre, d'une part, la forme du discours et, d'autre part son poids dans la causalité historique.

\footnotetext{
${ }^{34} \mathrm{Ni}$ même à un individu issu de la foule, cf. le long discours indirect prêté à Percennius en 1.17.

35 «Quippe ultro metum intentabat 〈uxor〉, multosque adstitisse libertos ac seruos qui eadem uiderint (...)». Ce discours entre dans la catégorie des discours indirects féminins instables, dont nous parlerons plus bas pour Poppée et Agrippine.
} 
Mais une telle conclusion ne rend pas compte, pour ces femmes sans réel pouvoir politique, de la plus grande partie des discours indirects, qui ne se concrétisent pas en action. Dans ces cas-là, la place importante réservée à la parole féminine par le recours au discours direct peut s'expliquer autrement : il s'agit de mettre en valeur une parole qui se signale par son exemplarité. Devenant un bonum exemplum, la voix féminine mérite alors de résonner plus directement dans l'histoire, et le choix du discours indirect (voire direct) se fait presque didactique. La majorité (plus des deux tiers) des discours indirects sans conséquence des non-Julio-Claudiennes sont des discours exemplaires : on y trouve essentiellement des femmes qui affirment à leur mari qu'elles les suivront dans la mort (comme Plancine en 3.15 et Pauline en 15.63), ou des femmes qui résistent à la torture sans trahir leurs proches (une esclave d'Octavie en 14.60, Epicharis et la conjuration de Pison en 15.57, auxquelles l'on peut ajouter l'intéressante répartie d'une Ligurienne face aux rapines des Othoniens en $H, 2.13$ ) : dans ces deux cas, c'est la valeur fides qui se trouve exemplifiée. La plus grande partie des paroles féminines exemplaires sont rapportées au discours indirect : ce n'est pas tant leur importance dans la consécution des événements que leur poids dans le discours du moraliste qui le justifie - ce qui explique que l'on puisse trouver dans ce corpus un nombre non négligeable d'esclaves, d'affranchies ou de femmes du peuple et de provinciales.

La forme même de ces discours indirects exemplaires est significative. Il s'agit en effet pour la plupart de discours très brefs (une ou deux propositions de niveau 1), bien encadrés par le récit avec, à leur gauche, un terme renvoyant à la locutrice, et à leur droite, un verbe de parole - en d'autres termes d'un discours fort contrôlé : "ipsa <Plancina> // sociam se cuiuscumque fortunae et si ita ferret comitem exitii // promittebat» (3.15); " una <ancilla Octauiae> // castiora esse muliebria Octauiae // respondit» (14.60); "illa 〈Paulina> contra // sibi quoque destinatam mortem // adseuerat» (15.63) ; «femina Ligus (...) uterum ostendens // latere // respondit » $(H, 2.13)$. Par leur constantia (le terme est utilisé pour la Ligurienne en $H, 2.13$ ), ces femmes obtiennent le droit de faire entendre leur voix dans le récit historique, mais précisément de faire entendre une voix clairement et nettement subordonnée à la narration, et non un discours indirect instable comme certaines Julio-Claudiennes (Poppée notamment), une voix devenue un outil idéologique pour l'historien. Thématiquement, ces discours indirects exemplaires se définissent par le refus général du pathétique ${ }^{36}$ comme objet du discours (même s'ils suscitent le pathétique chez le lecteur ${ }^{37}$ ), par une forme de détachement presque stoïcien face à la mort. Il est très intéressant que le seul «vrai » discours direct d'une femme autre qu'Agrippine, celui de Servilia, fille de Barea Soranus, soit non seulement un discours exemplaire, marquée du sceau de la piété filiale et du respect des institutions romaines, mais aussi un discours qui ne devient direct qu'une fois que le personnage féminin a renoncé au lexique pathétique : "primum strata humi longoque fletu et silentio, post... inquit » (16.31) : l'opposition primum... post indique bien cette évolution et le silentium central marque la césure entre les deux états. C'est ainsi la dignité de Servilia, qui quitte la posture pathétique, qui justifie qu'on ménage une place aussi exceptionnelle à sa parole. Le reste du discours, marqué par l'anaphore de nullus, développe très clairement l'ethos fort vertueux de la locutrice.

Ce parcours narratologique dans les discours des femmes théoriquement dépourvues de pouvoir politique montre bien que Tacite réserve à leur voix un espace presque autonome dans le récit que de manière exceptionnelle: lorsqu'elles sont assez importantes pour influencer le cours des événements, ou quand elles se muent en un outil idéologique pour le moraliste - encore faut-il mentionner que cela se fait alors dans des cadres discursifs très encadrés, très contrôlés. À l'inverse, nombre de femmes vertueuses ne s'expriment jamais chez Tacite, comme Galeria, la femme de

\footnotetext{
${ }^{36}$ Qui va parfois jusqu'au refus même de la parole : voir le comportement très clairement positif de la fille de Ségeste en $1.57:$ : neque uicta in lacrimas neque uoce supplex».

37 Il s'agit dès lors, selon la définition très classique de Marmontel, Éléments de Littérature, d'un pathétique réfléchi (ou indirect) et non direct, lequel consisterait à représenter les signes extérieurs du pathos (c'est le cas des lamenta des groupes féminins dont il a été question plus haut).
} 
Vitellius, ou même sa mère Sextilia, qui ne parle qu'en une occasion, précisément pour livrer un exemplum de modestie ${ }^{38}$.

La même analyse narratologique ne saurait convenir pour les discours des Julio-Claudiennes. Celles-ci sont, de fait, dans une position où la majorité de leurs interventions orales sont suivies d'action, comme on a pu le voir plus tôt ; par ailleurs, Tacite les représente comme des êtres passionnels, des «superbes » ou des «impudiques » pour reprendre les expressions de J.-M. Engel ${ }^{39}$, bien peu susceptibles de servir d'exemples éthiques. La double problématique de l'efficacité et de l'exemplarité du discours s'adapte donc mal à ce corpus. Néanmoins, une étude spécifique, pour les cinq grandes Julio-Claudiennes (Livie, Agrippine l'Aînée, Messaline, Agrippine la Jeune, Poppée) se révèle autant voire plus intéressante, en ce que le type (narratologique) de discours que Tacite leur attribue semble refléter le rapport au pouvoir particulier à chacune d'elles. Privées constitutionnellement et institutionnellement de l'imperium, ces femmes Julio-Claudiennes l'exercent néanmoins indirectement [référence biblio?], et leurs discours textualisent ou prolongent leurs convictions quant à l'exercice du pouvoir.

Si l'on étudie l'ensemble des paroles des cinq principales Julio-Claudiennes selon deux critères, le type de discours et son efficacité, les variations de résultats sont pour le moins surprenantes. Ainsi, Livie s'exprime dans les Annales quasiment toujours avec des discours narrativisés ( $90 \%$ des occurrences), et sa parole est majoritairement suivie d'effets ( $80 \%$ des cas). En cela, elle est suivie de près par Messaline, chez qui le discours narrativisé occupe $4 / 5^{\text {ème }}$ des interventions orales, mais qui est bien moins fréquemment la cause d'une action (2/5). Poppée s'exprime rarement (seulement trois discours), mais à chaque fois de manière imposante (ce sont trois discours indirects, et trois discours particulièrement conséquents, entre six et onze propositions infinitives) et efficacement. Agrippine l'Aînée et surtout Agrippine la Jeune ont une parole fréquente (respectivement six et onze interventions) et les formes de leur discours sont fort variées, la seconde étant d'ailleurs la seule à s'exprimer à la fois en discours direct, indirect, et narrativisé.

Ces différentes façons de faire entendre sa voix au sein du récit historique sont symboliques, nous semble-t-il, de différentes façons d'exercer une influence dans le cours des événements dans la Rome impériale, essentiellement taillée pour le pouvoir masculin : c'est là la fameuse ambition qui, souvent positive pour les hommes, devient un vice proprement féminin chez Tacite. Ainsi, que le discours de Livie soit essentiellement narrativisé, c'est-à-dire réduit à sa dimension événementielle, et pourtant particulièrement puissant, est symbolique de son rôle de femme de pouvoir de l'ombre, dont l'influence conséquente sur Auguste et sur Tibère ne vise jamais à la publicité : son refus d'apparaître aux funérailles de Germanicus ${ }^{40}$, sa complaisance aux arcana $^{41}$, son action "in occulto » (2.77) dessinent la cohérence de ces discours, qui semblent parfois presque anecdotiques, mais qui constituent souvent en réalité le moteur d'une décision impériale : son comportement pour protéger Plancine en est le meilleur exemple (cf. supra). Le fait que la plupart de ses interventions soient mentionnées à l'ablatif de cause ${ }^{42}$ nous paraît aller exactement dans le même sens : elles ont l'apparence de circonstants, mais sont plutôt à l'origine de bien des actions.

\footnotetext{
${ }^{38} H, 2.64$ : Galeria constitue le modèle de la femme d'empereur, en ce qu'elle ne se mêle pas aux affaires politiques («non immixta tristibus»). Le chapitre du Dialogue des Orateurs consacrées aux exempla maternels de l'époque républicaine évoque les actions vertueuses de Cornelia (mère des Gracques), d'Aurelia (mère de César) et d'Atia sans leur donner une seule fois la parole. Même constat pour les vertueuses Germaines (Germ., 19, J.-M. Engel, 1972, p. 297bis).

${ }^{39}$ J.-M. Engel, 1972, p. 300.

${ }^{40}$ "Tiberius atque Augusta publico abstinuere », 3.3.

${ }^{41}$ Cf. notamment la leçon que Sallustius lui donne à propos de la divulgation des informations en 6.3. Symboliquement, ce n'est qu'après coup que, bien souvent, on reconnaît l'influence de Livie, comme en creux (voir par exemple après sa mort, 5.3 : «litterae <Tiberii> quas pridem adlatas et cohibitas ab Augusta credidit uulgus»).

42 "Hortatu», 1.3 ; « secretis Augustae precibus », 3.15 ; "precibus uxoris », 4.57 ; «precibus Augustae», 6.26 .
} 
Agrippine l'Aînée, elle, incarne un rapport au pouvoir plus public, comme l'attestent ses trois discours indirects (sur six interventions orales), mais aussi une parole rapidement privée de poids avec la mort de Germanicus, et qui n'a jamais une efficacité indubitable et concrète : très symboliquement, les trois passages où il est fait état d'une possible puissance de son discours sont aussi les trois passages où sa parole est mentionnée à travers celle d'autrui, et d'un homme de surcroît - Domitius Celer qui craint ses planctus (2.77) ou Tibère qui soupçonne ses preces (4.17); même son activité auprès de Germanicus en Germanie, notamment lorsqu'elle adresse «des éloges et des remerciements » («laudes et grates », 1.69) aux légions, est évoquée par la médiation de Pline l'Ancien, un des auctores de Tacite ${ }^{43}$. La puissance du discours d'Agrippine, très différent par sa publicité assumée de celui de Livie, ne s'actualise jamais réellement, probablement à cause de la mort précoce de son mari et du pouvoir impérial fort de Tibère ${ }^{44}$; en outre, elle se trouve rapidement réduite au rôle de miserrima coniux (2.70), incompatible avec celui de femme de pouvoir.

Si Agrippine pouvait incarner un projet de communication différent de celui de Livie, Messaline, de manière surprenante, se révèle plus proche à cet égard de la mère d'Auguste. Comme pour elle, ses discours sont majoritairement des discours narrativisés, qui symbolisent une action discrète mais puissante, du moins avant sa disgrâce. Sa communication ne reflète en aucun cas la frénésie de ses débauches, mais relève bien plutôt d'une parole simple et directe, comme l'indique sa toute première intervention dans ce qui a survécu des Annales («monet », 11.2). La progressive perte d'efficacité de sa voix, qui revient à des pratiques plus féminines à la fin, est peut-être la conséquence de l'incompatibilité entre cette conception d'un pouvoir féminin fort mais discret, quoique assumé, et sa vie privée, dont les historiographes ont rendu célèbres les excès. Chez Messaline coexistent en effet un caractère essentiellement féminin (c'est-à-dire passionnelle, impudique, selon la conception tacitéenne), et un pouvoir presque masculin ${ }^{45}$, notamment dans le début du livre XI, et son discours reflète exactement cette hésitation ${ }^{46}$ : ainsi, lors du procès d'Asiaticus, ses larmes pour l'accusé ("Messalinae quoque lacrimas exciuit», 11.2), qui la rattachent au pathétique féminin, ne l'empêchent pas d'ordonner son incarcération ( «monet Vitellius ne elabi reum sineret »).

Agrippine la Jeune, nous l'avons dit, reste le personnage féminin le plus complet dans ses interventions orales ; c'est la seule de ce groupe qui s'exprime au discours direct. Les nombreuses variations entre le discours narrativisé, le discours indirect et le discours direct expriment peut-être à quel point la fille de Germanicus est à l'aise dans l'exercice du pouvoir ; surtout, l'évolution de la forme de ces discours reflète l'évolution de son parcours "politique ». Son influence naissante sur Claude est d'abord textualisée par des discours narrativisés efficaces (impetrare utilisé en 12.8 et 12.27), et son pouvoir grandissant se développe alors que sa voix prend plus d'importance dans le récit historique, passant du discours narrativisé au discours indirect. Avec l'adoption de Domitius Néron par Claude (12.25), elle est alors clairement liée en deux points au pouvoir (femme de l'empereur, mère du prince), et sa parole atteint un pic d'efficacité, symbolisé par l'omniprésence de sa voix : trois interventions en à peine deux chapitres ${ }^{47}(12.41$ et 42$)$, dont deux au discours indirect, et toutes suivies d'effets immédiats. L'émancipation de Néron, à partir du début du livre XIII, va de pair avec la déchéance d'Agrippine et de son discours, qui, très symboliquement, se réduit dans ce début du livre XIII à des discours narrativisés inefficaces ${ }^{48}$. Cette chute n'est pas linéaire, comme le prouve le sursaut

\footnotetext{
${ }^{43}$ «Tradit C. Plinius, Germanicorum bellorum scriptor, stetisse apud principium pontis laudes et grates reuersis legionibus habentem ».

${ }^{44}$ Peut-être aussi à cause du fait qu'elle a construit son discours et son pouvoir hors de Rome, cf. R. Hälikkä, 2002 , p. 100.

${ }^{45}$ Sa mort (11.37-38) est extrêmement symbolique à cet égard, Messaline hésitant à se suicider et finissant par se faire abattre sans aucune dignité par le tribun que Narcisse a envoyé.

46 Très symbolique aussi, le fait que Messaline hésite entre lieux publics et lieux privés pour exhiber son pouvoir, cf. H. Hälikkä, 2002, p. 97-99.

47 Alors même que Tacite ne consacre à ce moment du livre XII que trois chapitres (12.41-43) aux affaires romaines, prises entre d'une part les troubles en Bretagne (12.31 à 40) et les guerres au Proche-Orient (12.4452). On mesure bien ici l'omniprésence de la voix d'Agrippine.

${ }^{48}$ Sa première intervention dans ce livre XIII est d'ailleurs intégrée dans un ablatif absolu à valeur adversative : « quod quidem aduersante Agrippina » (13.5).
} 
du long discours direct de 13.21 qui est immédiatement suivi d'effet ; mais il s'agit alors d'une posture défensive, et qui n'est guère plus que le lointain écho de sa puissance passée, comme peut l'indiquer l'expression «ferociae memor» qui introduit sa plaidoirie. Sa parole devient purement exemplaire avec les discours directs rapportés au moment de sa mort.

Poppée vient occuper très nettement l'espace discursif laissé vacant non par la mort d'Agrippine, mais par sa chute et son aphonie, dès le milieu du livre XIII (et à l'exception de ses ultima uerba). Trois interventions sont à créditer à Poppée, total assez faible en fin de compte, mais disséminées à intervalles réguliers $(13.46 ; 14.1 ; 14.61)$. Poppée parle donc peu, mais efficacement ses discours aboutissent toujours à une décision de la part de Néron - et massivement. Ce sont trois discours indirects très longs, et tous adressés à Néron : comme Livie avec Auguste et Tibère, et à la différence de Messaline et d'Agrippine la Jeune avec Claude, le pouvoir féminin ne s'exerce avec elle qu'indirectement, par influence, à travers l'autorité de l'empereur, et sans se substituer à elle. Néanmoins, Poppée semble faire une plus grande publicité de sa parole, bien moins discrète que celle de Livie (sa voix n'est pas narrativisée). Surtout, ce qui marque dans ces discours reste leur instabilité : en 14.1 et 14.61, le discours indirect n'est absolument pas introduit par un verbe de parole et apparaît presque inopinément dans le récit ${ }^{49}$. Ailleurs, en 13.46, le discours n'est pas strictement rattaché à un moment particulier, mais semble délayé dans le sommaire qu'effectue alors le récit. Ses paroles sont d'ailleurs introduites par un fréquentatif ( "nuptam esse se dictitans... »), mais la longueur et le détail du discours prouvent bien qu'il ne peut s'agir de mots simplement répétés à de nombreuses reprises. Comment analyser cette instabilité ? Peut-être est-elle le signe du rapport au pouvoir particulièrement insidieux que met en jeu, selon Tacite, le personnage de Poppée. De même que son discours indirect s'insinue de façon inattendue dans le récit, de même le personnage se sert de ses charmes pour, à travers Néron, influencer - de façon scandaleuse pour l'historien-moraliste - le cours des événements et le destin de l'Empire.

Cette forme de discours instable ne se trouve pas uniquement pour Poppée, même si c'est elle qui l'illustre le plus parfaitement. Le recours assez fréquent à l'infinitif de narration pour introduire des paroles féminines, au-delà même des Julio-Claudiennes, abonde dans le même sens. De fait, l'emploi de l'infinitif, sans entrer dans le détail, suppose bien que l'on vide l'action de toute marque temporelle ${ }^{50}$, et qu'on la rattache à une temporalité extrêmement floue ${ }^{51}$. La même analyse vaudrait pour les fréquentatifs, qui inscrivent des développements oratoires souvent tout à fait circonstanciés dans une temporalité itérative. Dénier à un discours qu'il ait pour cadre énonciatif un moment précis et unique, c'est en fin de compte dénigrer sa portée. Il est intéressant de constater que cette technique touche en majorité (quoique non exclusivement) les discours féminins et les discours de la foule : le verbe fremere employé comme infinitif de narration, par exemple, est appliqué à un discours d'Agrippine en 13.13 (c'est le fameux «muliebriter fremere »), mais aussi pour les paroles de la foule en 11.28 et en $H, 2.6$ (pour ne relever que les infinitifs de narration); ce terme particulièrement significatif opère du même coup une réduction au matériau sonore de ce qui demeure de véritables actes rhétoriques. C'est que, pour la femme comme pour la foule, Tacite a conscience d'un paradoxe : ces entités sociales, autrefois exclues du pouvoir politique, sont en train, à son époque, d'y accéder, par des voies détournées, et leur voix porte de plus en plus ; refusant de reconnaitre ostensiblement la nouvelle puissance de leur discours, l'historien réussit pourtant à en rendre compte au cœur de son projet historiographique avec des formes discursives symboliquement paradoxales.

\section{CONCLUSION}

\footnotetext{
${ }^{49}$ C'est très net en 14.61: «[Poppaea] semper odio, tum et metu atrox (...), prouoluta genibus eius <sc. Neronis >, non eo loci res suas agi (...)».

${ }^{50}$ A. Orlandini, «Infinitif exclamatif et infinitif de narration », 2002, notamment p. 198 : « la prédication (...) est focalisée, dépourvue de tout ancrage temporel déictique ».

${ }^{51}$ S. Adema, "Temporal bases and the use of the narrative infinitive in the Aeneid”, 2007, p. 9.
} 
Parler d'« opinion nuancée » de Tacite à l'égard des femmes, comme le fait É. Aubrion ${ }^{52}$, nous paraît très juste : la complexité de leur discours et de la façon dont il est représenté dans le récit historique en est la meilleure preuve. féminins,

Pour synthétiser nos remarques, concernant la parole des Julio-Claudiennes ${ }^{53}$ : par les discours

$\Rightarrow$ Tacite peut peindre les empereurs Claude et Néron comme des faibles et peindre Tibère comme mené par la crainte (irraisonnée, face à Agrippine), caractéristique du tyran ;

$\Rightarrow$ pour Messaline et Octavie : la question de l'efficacité fait ressortir les caractéristiques du personnage $($ Messaline $=$ transgression $:$ Octavie $=$ victime $)$;

$\Rightarrow$ Agrippine II : le plus ambigu, le plus riche ; en raison de sources Mémoires d'Agrippine?

$\Rightarrow$ efficacité à mettre en relation avec les menaces.

Il y a donc un véritable travail de Tacite, centré sur les femmes, certes présentées de manière négative, mais qu'il a utilisées comme outil de définition du régime impérial.

Concernant les autres femmes et l'étude narratologique de tous les discours féminins :

$\Rightarrow$ les femmes hors de la domus ont finalement peu de pouvoir, mais une partie de celui-ci vient de leur capacité à émouvoir le pathétique, notamment dans la foule ;

$\Rightarrow$ la narratologie pourrait montrer que la forme des discours des non-Julio-Claudiennes permet de distinguer certaines paroles pour leur importance dans le cours des événements ou pour leur exemplarité ;

$\Rightarrow$ mais que, pour les Julio-Claudiennes, les différents types de discours sont surtout l'écho de conceptions du pouvoir différent, et qu'à ce titre, les structures du récit de Tacite reflètent les structures socio-politiques de l'époque impériale ${ }^{54}$.

Conclusion générale : l'ambiguïté, notamment politique, de la pensée tacitéenne vaut aussi pour les femmes $^{55}$ : il faudrait constamment distinguer la forme (ostensiblement négative) du fond, les femmes ayant finalement un rôle complexe, que ce soit au niveau historique (omniprésence et puissance des JC) ou au niveau historiographique. Le débat à propos des femmes qui se tient au Sénat reste d'ailleurs un débat antilogique (3.33-34), avec des arguments pro et contra : quelle qu'ait été son opinion, Tacite a su garder les deux faces de la question dans son œuvre.

\footnotetext{
${ }^{52}$ É. Aubrion, Rhétorique et Histoire chez Tacite, 1985, p. 667.

${ }^{53}$ Une grande absente, Antonia : aucune parole? Alors que importance, cf I. Cogitore, « Formes d'opposition sous Caligula, le rôle des femmes » dans Lo spazio del non allineamento a Roma fra tarda Repubblica e primo principato, Forme e figure dell'opposizione politica, R. Cristofoli, A .Galimberti, Fr. Rohr Vio, 2014, 167-181. ${ }^{54} \mathrm{~L}$ 'on reprend ainsi le vocabulaire sociocritique, à laquelle la méthodologie de cette partie narratologique était finalement affiliée (cf. l'article d'introduction à la sociocritique de R. Robin, « De la sociologie de la littérature à la sociologie de l'écriture ou le projet sociocritique », Littérature, 70, p. 99-109).

${ }^{55}$ R. Hälikkä, 2002, p. 78, qui affirme que l'ostensible misogynie de Tacite cache un intérêt profond pour la figure de la dux femina. Le même type de constat vaut pour les foules.
} 